\title{
The modern Forel-Ule scale: a 'do-it-yourself' colour comparator for water monitoring
}

S. Novoa

Stefani.Novoa@nioz.nl

M. R. Wernand

H. J. van der Woerd
NIOZ Royal Netherlands Institute for Sea Research, NL-1790 AB Den Burg, the Netherlands

NIOZ Royal Netherlands Institute for Sea Research, NL-1790 AB Den Burg, the Netherlands
Institute for Environmental Studies (IVM), VU University Amsterdam, De Boelelaan 1087, 1081 HV Amsterdam, the Netherlands

The colour comparator Forel-Ule scale has been used to estimate the colour of natural waters since the 19th century, resulting in one of the longest oceanographic data series. This colour index has been proven by previous research to be related to water quality indicators such as chlorophyll and coloured dissolved organic material. The aim of this study was to develop an affordable, 'Do-it-Yourself' colour scale that matched the colours of the original Forel-Ule scale, to be used in water quality monitoring programs by citizens. This scale can be manufactured with high-quality lighting filters and a white frame, an improvement with respect to the materials employed to manufacture the original scale from the 19th century, which required the mixing of noxious chemicals. The colours of the new scale were matched to the original colours using instrumental and visual measurements carried out under controlled lighting conditions, following the standard measurement protocols for colour. Moreover, the colours of the scale are expressed in Munsell notations, a standard colour system already successfully used in water quality monitoring. With the creation of this Modern Forel-Ule scale, as a 'Do-it-yourself' kit, the authors foresee a possible use of the Forel-Ule number as a water quality index that could be estimated by means of participatory science and used by environmental agencies in monitoring programs.

[DOI: http://dx.doi.org/10.2971/jeos.2014.14025]

Keywords: Forel-Ule scale, water quality, participatory science, ocean colour, colourimetry, citizen science, water monitoring

\section{INTRODUCTION AND PROBLEM STATEMENT}

The colour of natural waters has been measured globally and intensively by oceanographers and limnologists since the $19^{\text {th }}$ century by means of the Forel-Ule (FU) colour comparator scale [1]-[3], resulting in one of the longest oceanographic data series after the Secchi disk depth. Wernand et al. (2013) [4] used these data sets to estimate global changes occurring in the ocean in relation to the chlorophyll-a concentration, a key index of phytoplankton biomass and primary productivity studies [5]. It was also shown that the FU scale is related to coloured dissolved organic matter (CDOM) absorbance, considered as well to be one of the water quality indicators [6].

The colour of natural waters is mainly affected by three types of elements: 1) The pigments in organic material, normally expressed as chlorophyll-a concentration (mainly of greenish colour unless it is a specific type of algal bloom), 2) CDOM, an indicator of freshwater content and plant degradation (also known as yellow substance), and 3) Total Suspended Material concentration (TSM), an indicator of turbidity that is influenced by coastal erosion, riverine flux, and wind- or currentgenerated re-suspension (usually of brownish or grey colour depending on location). Therefore, the colour of water is an apparent optical property that provides information on its composition, and for that reason, the concept of 'optical water quality' was introduced by Kirk (1988) [7], who highlighted the importance of measuring these optical properties for water quality monitoring.

Colour, together with clarity (or transparency), is one of the first apparent features to the human eye when observing natural waters. Humans associate pristine water bodies with deep blues or turquoise colours and a certain depth visibility is necessary to safely enjoy water activities (Bathing water directive 76/160/EEC Report). Hence, colour strongly affects the visual and aesthetic perception of the public and their recreational use $[8,9]$. Moreover, the colour of water has a strong effect on aquatic ecosystems, as it has been shown to affect photosynthesis and primary productivity $[10,11]$, predation regimes of herbivores [12], invertebrate behavior [13, 14] and alter the availability and toxicity of heavy metals to fish $[15,16]$.

Changes in colour and clarity in aquatic systems can be caused by natural causes, such as heavy rains that transport organic material, nutrients and mineral. However, these changes can also be due to anthropogenic activities such as the introduction of an excess of nutrients, originating from fertilisers used in agriculture. These inputs can then cause algal blooms that change the colour and clarity of the water, a phenomenon known as eutrophication, which is a major environmental issue across Europe [17]. In order to determine 
if a change in colour is due to a particular anthropogenic activity, it is important to collect long-term data on the colour and clarity of water bodies (British Columbia Ministry of Environment) [18], making it necessary to have easy-to-use and affordable tools to cover large areas, as well as a high sampling frequency.

Clarity of natural bodies can be easily and affordably estimated by means of a Secchi disk [19, 20]. However, colour is a more difficult feature to measure as it requires the analysis of the wavelength distribution of light. This analysis can be achieved using a spectroradiometer [21], but this type of instrument is expensive and not suitable for citizen monitoring surveys and fast interpretation. Davies-Colley [22, 23] showed that a colour-matching method using the Munsell system [24, 25], one of the most widely known colour systems, is suitable for routine water resources surveys and monitoring, since humans can easily match colours observed simultaneously. This method was implemented by the National Institute of Water and Atmospheric Research (NIWA) as part of the water quality monitoring programs [26]. However, the matching of natural colours to the Munsell scale is a relatively new technique and it seems to be employed only in some water quality monitoring programs [8, 27] (New Zealand, British Columbia), providing a limited amount of data for global long-term studies.

The original FU colour comparator scale consists of 21 coloured solutions, between indigo blue and cola brown, going through green. These solutions, made with distilled water, ammonia, copper sulfate, potassium-chromate and cobalt-sulfate, are placed in vials. The colour is determined by comparison of the colour of the water observed above a Secchi disk to the coloured vials. Although easy to use, this scale is not simply reproduced [28], and furthermore the chemicals used to prepare the solutions can be toxic to humans [29].

The colour of natural waters and other materials depends on three main features: hue, saturation and brightness. Hue refers to how the colour is described (e.g. 'blue' or 'green'), and it is determined by the dominant wavelength in the visible spectrum. Saturation or colour purity (less saturated is more greyish than saturated, which has a more intense colour) depends on the spread of energy around the dominant wavelength $[30,31]$. Brightness refers to the amount of energy detected by the human eye, which is most sensitive around the green wavelength $(555 \mathrm{~nm})$. The FU scale involves the matching of only the hue, while the Munsell system considers purity and brightness as well.

Framed within the European Project CITCLOPS (Citizens' Observatory for Coast and Ocean Optical Monitoring), the aim of this manuscript is to present a tool that can be employed by citizens to determine the colour of the water based on the FU colour comparator scale. A scale that accurately matches the original FU colours was developed using accessible and affordable materials. Hence, this 'Do-It-Yourself' kit can be prepared using high-quality illumination filters and a frame made of a white Plexiglas (or other white material). The intention was to create a colour comparison tool that could be used

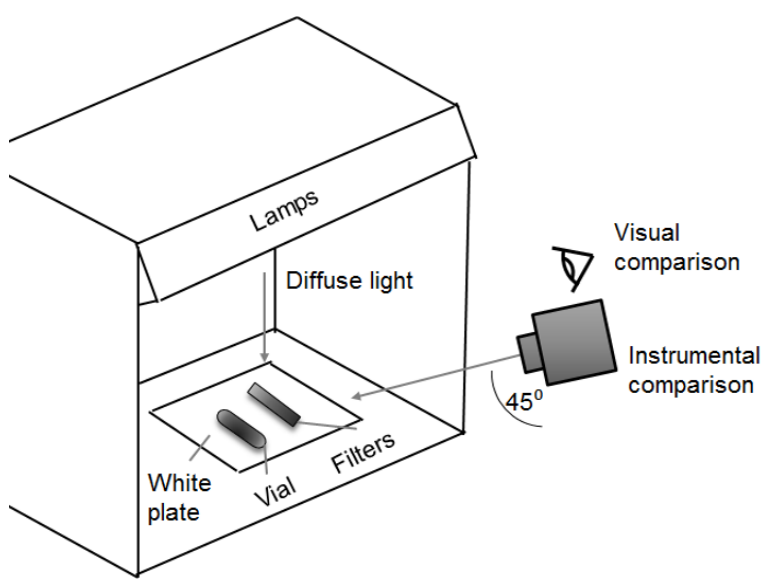

FIG. 1 Light cabinet set-up for the colour-matching procedure. The coloured vials and filters (combinations) were placed flat, at the bottom of the cabinet, on top of a white plate side by side, $4.0 \mathrm{~cm}$ apart. Comparisons were carried out under a $45^{\circ} /$ normal geometry, and the lamps (D65, D75) were placed above the objects at a distance of 50 $\mathrm{cm}$.

by anyone willing to participate in environmental monitoring, providing an easy method to record not only the colour, but also the clarity of natural waters, since this scale needs to be used together with a Secchi disk. In addition, the colours of the scale were matched to the colours of the Munsell system, providing a connection with additional work conducted on monitoring of natural waters using colour-matching techniques [22], and combining the advantages of both scales to work towards an improved colour estimation technique for water quality monitoring.

\section{MATERIALS AND METHODS}

\subsection{Visual and instrumental colourimetric measurements}

In this study we compared the colour of two objects by means of visual inspection and using a spectrometer in a dedicated laboratory environment. The visual assessment of a colour by an observer is as important as the colour measured by the instrument, especially in our case where the colour-matching of the water is going to be assessed visually. The colour appearance of an object is affected by many factors, including the spectral properties of the light source, the level of illumination, background reflectance and the object composition. Hence, to correlate the instrumental measurements with our visual measurements, the conditions under which the evaluation is conducted need to be very restricted [32]. For that reason, controlled lighting conditions were prepared in a light cabinet placed in a dark room (Figure 1). To ensure the correctness of the visual assessments, the authors of this document successfully passed the Farnsworth-Munsell 100 Hue Colour Vision Test [33] (www.colour-blindness.com, search for: farnsworth munsell 100-hue colour vision test), a test commonly used to determine any colour vision deficiencies.

\subsection{Colour-matching procedure}

The liquid colours of the FU scale were replaced by plastic colour-effect lighting filters of the brands LEE 
(www.leefilters.com) and Roscolux (www.rosco.com). Visual and instrumental (spectral measurements) matches of the filters against the coloured liquids in vials were conducted under diffused artificial daylight simulators D65 (VeriVide, width: $600 \mathrm{~mm}, 18 \mathrm{~W}$ ) and D75 (VeriVide, width: $600 \mathrm{~mm}$, $20 \mathrm{~W})$, on top of a diffuser, in a colour assessment cabinet (VeriVide) with a grey coating inside (Munsell num. 5). The term illuminant refers to a spectral power distribution realizable by a light source, while the term source refers to any light emitting object (relative energy distribution in the visible spectrum, $380-750 \mathrm{~nm}$ ), such as the sun or a light bulb.

The D65 light source has a correlated colour temperature of $6500 \mathrm{~K}$ and was selected because it represents the average daylight, at various times throughout the day and throughout the year. According to the International Commission on Illumination (CIE, www.cie.co.at), the D65 illuminant should be used in all colorimetric calculations requiring representative daylight, unless there are particular reasons to use a different illuminant. The D75 light source was chosen as well because it represents the North Sky daylight with a correlated colour temperature of $7500 \mathrm{~K}$ (slightly bluer than D65) and has been related to overcast sky conditions [34]. The D65 and D75 CIE Standard Illuminant defined by the CIE are part of the D series of illuminants that try to describe standard illumination conditions at open-air in different parts of the world. Hence, these two illuminants were selected to obtain a matching filter scale under different open-air lighting conditions.

The colour-matching procedure was conducted following the recommendations of the American Society for Testing and Materials Standard International publications (ASTM E1164 12, ASTM E179-12; ASTM E308) [35]-[37], commonly used in the industry to assess the colour differences between batches of manufactured objects. The liquid vial and the corresponding combination of colour effect filters were placed at the bottom part of the lighting cabinet, lying flat over a white plate. Both the visual and instrumental comparisons were carried out at a $45^{\circ}$ degree angle, labeled as $45^{\circ} /$ normal geometry by the CIE [36] (International Commission on Illumination, ASTM E179-12) and the lamps (D65, D75) were placed above the objects at a distance of $50 \mathrm{~cm}$. The visual comparison was conducted by observing simultaneously both the vials and the filters, placed side by side, $4.0 \mathrm{~cm}$ apart. The instrumental measurement was conducted using a PHOTORESEARCH PR-655 SpectraScan spectroradiometer (www.photoresearch.com). The device was placed in front of the cabinet, with the samples centered at the bottom and at an angle of $45^{\circ}$ with respect to the samples, at the same position as the one used for the visual comparison (see Figure 1). The measurements were carried out always with the samples placed at the same location inside the cabinet, since slight differences in the chromaticity values calculated for the light source were observed in different parts of the cabinet. Also, the light source irradiance was measured every single time the matching procedure was conducted, as minor differences in the intensity were observed as well. The lamp irradiance was measured at the beginning and the end of every measurement session, using a PHOTORESEARCH PR-640 spectrometer with a cosine collector. The matching procedure

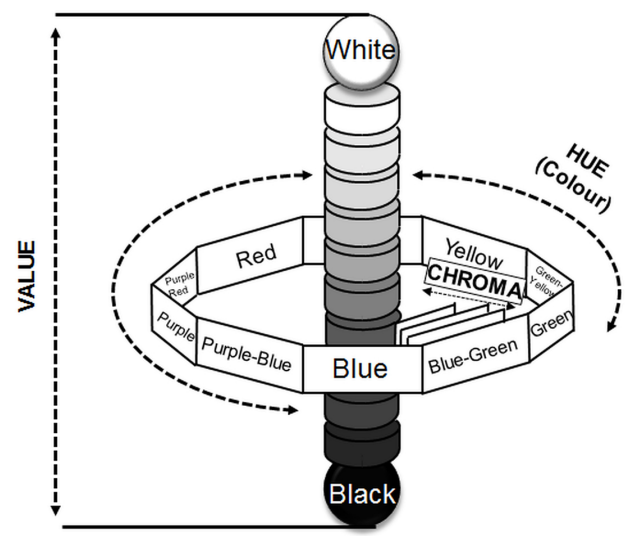

FIG. 2 Munsell color system diagram showing the Hue, the Value and the Chroma.

started with a visual selection of the filters from the samples according to the transmission curves provided by the manufacturer, and the observed colours of the filters, followed by the spectral match using reflectance measurements of both the vials and filter combinations. The 'SpectraWin2' software from PHOTORESEARCH was used to visualise and calculate reflectance spectra, chromaticity coordinates, saturation and the dominant wavelength of the measured samples.

The association with the Munsell system (Figure 2) was conducted visually under the D65 light source using the glossy version of 'Munsell book of colors' [25] (www.munsell.com). The samples from the book (commonly referred as 'chips') were matched visually to the FU coloured vials, one by one. The Munsell colour system notation consists of three independent dimensions hue $(\mathrm{H})$, value or lightness $(\mathrm{V})$ and chroma (C). The system is divided into five principal hues: Red, Yellow, Green, Blue, and Purple, along with 5 intermediate hues (e.g. BG, as Blue-Green). The value or lightness changes vertically along the hue, from black (value 0) at the bottom, to white (value 10) at the top. Chroma represents the "purity" of a colour (related to saturation), with higher chroma being more pure and lower chroma being paler colours.

\subsection{Calculations}

The SpectraWin 2 program calculated the reflectance, defined as the ratio of the measured radiance of the object and the irradiance of the lamp source. Dominant Wavelength (DW) is defined as the wavelength on the CIE diagram boundary (or colour locus) that, when mixed in appropriate proportions with the white reference, yields the chromaticity coordinate of the measured sample (Figure 3). Mathematically, a line is drawn from the standard illuminant, through the measured point to the monochromatic locus (boundary) of the CIE diagram and the dominant wavelength is where the line intercepts the locus (Figure 3). Saturation (or Purity) is the measure of how pure, or monochromatic, the colour is. The distance of the measured sample to the boundary at the dominant wavelength location is divided by the distance of the standard illuminant (achromatic point) to the same point. This measure is expressed in a percentage, $100 \%$ saturation is a perfectly saturated colour (lies on the monochromatic locus), and it is 


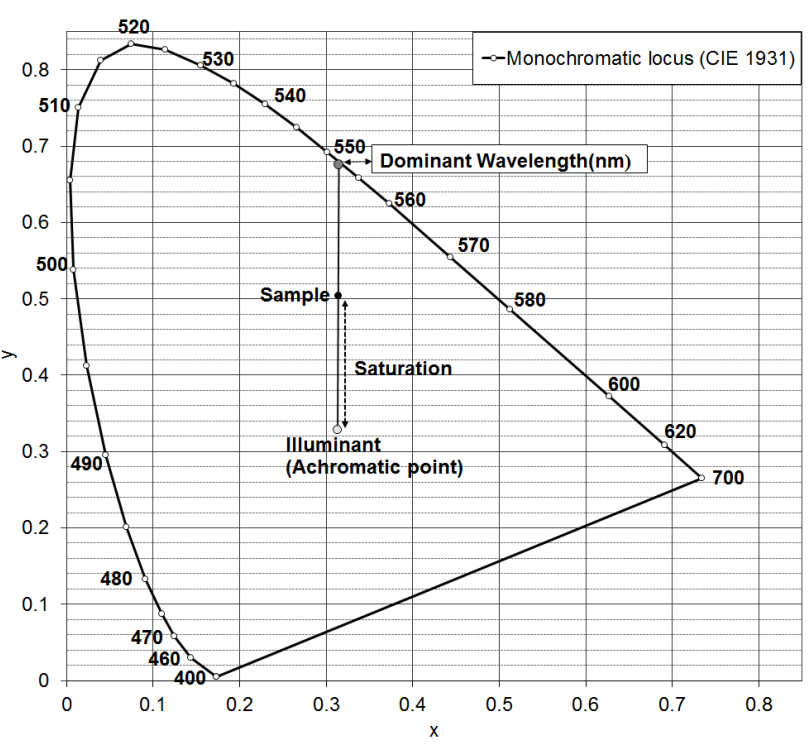

FIG. 3 The CIE1931 X, y chromaticity diagram for monochromatic colours. The outer curved boundary is the spectral or monochromatic locus, with wavelengths in nanometers. Each measured sample is defined by its $(x, y)$ chromaticity coordinate set and colour 'purity' or 'saturation'

calculated using the following formula:

$$
\text { Saturation } \%=\frac{T S_{d} * 100}{R I_{d}}
$$

Where:

$\mathrm{TS}_{d}=$ Distance from sample to monochromatic locus.

$\mathrm{RI}_{d}=$ Distance from standard illuminant to monochromatic locus.

The tristimulus values $X Y Z$ were calculated by the SpectraWin2 software, using the reflectance spectra and according to:

$$
\begin{aligned}
& X=683 \int_{360}^{830} S(\lambda) \bar{x}(\lambda) \Delta(\lambda) \\
& Y=683 \int_{360}^{830} S(\lambda) \bar{y}(\lambda) \Delta(\lambda) \\
& Z=683 \int_{360}^{830} S(\lambda) \bar{z}(\lambda) \Delta(\lambda)
\end{aligned}
$$

Where $S(\lambda)$ is the spectral data that depends on the wavelength $(\lambda), \mathrm{XYZ}$ are the tristimulus values and $\bar{x}, \bar{y}, \bar{z}$ are the colour-matching functions (CMFs). Lumens are converted to watts using the 683 constant.

The CIE 1931 chromaticity coordinates were calculated according to:

$$
x=\frac{X}{X+Y+Z} \quad \text { and } \quad y=\frac{Y}{X+Y+Z}
$$

More information on the calculation of tristimulus values and chromaticity coordinates can be found in Wyszecki and Stiles (1982) [38].

The differences between the colours of the vials and the filter combinations were estimated using the dominant wavelength and the saturation. From the visual inspection, we concluded that it was important to minimise the deviation in both the hue and saturation. The deviations between vials and filters had to be considerably smaller than the difference in dominant wavelength between adjacent filters. Thus, there was enough colour spacing between the filters to be able to discriminate among them, but also provide a range of error to account for the difference in colour appearance of the two types of materials compared, liquid in vials versus plastics. The deviation in saturation between vials and filters was also kept as low as possible. The relative dominant wavelength difference percent (DWD \%) and saturation difference (SD \%) percent of the filters $(f)$ with respect to the vials $(v)$ were calculated as follows:

$$
\operatorname{DWD} \%=\frac{D W_{v i}-D W_{f i}}{D W_{v i}-D W_{v j}}
$$

Where $D W_{v i}$ and $D W_{f i}$ are the dominant wavelengths measured for vial $v i$ and the filter combination $f i$ in nanometers $(\mathrm{nm})$, normalised to the difference in dominant wavelength between two adjacent FU vials $\left(D W_{v i}-D W_{v j}\right)$, with respect to the previous and the posterior adjacent FU vial $(v j)$, providing two values, the 'DWD + ' \% and 'DWD -' \%, respectively.

The difference in saturation percentage (SD \%) was calculated in the same manner, but normalised to the saturation of the $\operatorname{vial}\left(S_{v i}\right)$.

$$
\mathrm{SD} \%=\frac{S_{v i}-S_{f i}}{S_{v i}} \times 100
$$

Where $S_{v i}$ and $S_{f i}$ are the saturation values measured for the vial $i(v i)$ and the corresponding filter combination $(f i)$.

The exact Munsell notations were calculated using the program 'colour2drop' (developed by Zsolt Kovacs and available at www.ing.unibs.it/), based on the information on the Munsell calculations found in the American Society for Testing and Materials publication [39] (ASTM D 1535).

\section{RESULTS}

A total of 30 colour effect filters (27 LEE and 3 Roscolux) were necessary to match the colours of all 21 vials (vial numbers shown in Table 1). The colours measured (dominant Wavelength, DW, and saturation, S) of the filters deviated a maximum of $20.83 \%$ and $12.18 \%$, respectively, from the colours of the vials.

Figure 4 shows the comparison of the DW estimated for the FU vials and filters. There is a strong match in DW, between the vials and the filters, so the selected filters were considered to be appropriate for the scale. It can be noticed that the DW gradually increases up to FU7, after which there is a sharp upward slope until FU11. Then, the slope between FU11-FU21 becomes more moderate again. There is also a higher angle difference between adjacent FU colours in the 'bluer' part of the scale (FU1-11), with an average of $9 \mathrm{~nm}$, compared to the 'browner' part of the scale (FU12-21), with an average $3.8 \mathrm{~nm}$. The highest dominant wavelength difference (DWD) between the filters and the vial was obtained for FU6, with a $20.83 \%$ difference with respect to the previous adjacent colour FU5. 


\begin{tabular}{|c|c|}
\hline FU Number & Filter combinations \\
\hline 1 & L200 \\
2 & L501+L063 \\
3 & L504+ L201 \\
4 & L241+ L061 \\
5 & L504+L241 \\
6 & L503+ L730+ L242 \\
7 & L242 \\
8 & L278+R3316+L242 \\
9 & L733+L HT007+L730 \\
10 & L245+L HT007+L731 \\
11 & L731+R4515+R4560 \\
12 & L244+L223 \\
13 & L206+L244 \\
14 & L244+L443+L763 \\
15 & L244+L009+L298 \\
16 & L244+L223+L285+L298 \\
17 & L650 \\
18 & L223+L650+L206 \\
19 & L230+L763+L249 \\
20 & L230+L223+L506 \\
21 & L207+L506+L298 \\
\hline
\end{tabular}

TABLE 1 Filter combinations to obtain each FU colour. Abbreviations: $L=$ Lee filters; L HT = High temperature Lee filters; $R=$ Roscolux filters. The order of the filters is important, the first filter has to be placed at the top and the last at the bottom.

The next highest difference was obtained for FU13 (DWD+ = $16.83 \%)$.

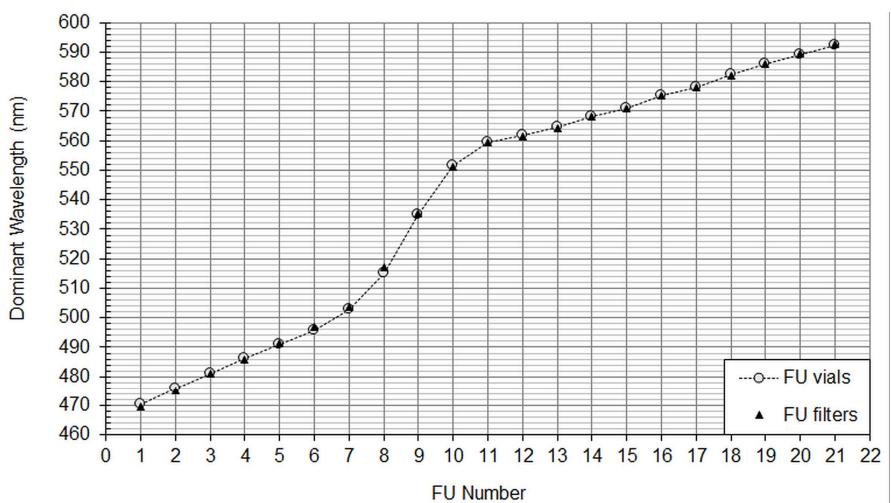

FIG. 4 Dominant Wavelength of the instrumentally measured vials and filter combinations.

Figure 5 shows the saturation percentage for both the vials and the filters. It can be noticed that FU1 has the highest saturation of all the FU colours, after which it decreases until FU8, where it increases again until FU11, and stays stable for the rest of the FU numbers. The highest difference in saturation (SD \%) was obtained for FU8, which showed a $12.18 \%$ less saturation of the filters' colours with respect to the vials. The rest of the FU colours showed a difference in saturation below $10 \%$, except for FU6 and FU12 (10.62 \% and $10.92 \%$, respec-

\begin{tabular}{|c|c|c|c|c|c|c|c|c|c|c|c|}
\hline FU Number & \multicolumn{9}{|c|}{ FU vials } \\
& $x$ & $y$ & $\begin{array}{c}\text { DW } \\
(\mathrm{nm})\end{array}$ & $\begin{array}{c}\mathrm{S} \\
(\%)\end{array}$ & $\mathrm{x}$ & $\mathrm{y}$ & $\begin{array}{c}\text { DW } \\
(\mathrm{nm})\end{array}$ & $\begin{array}{c}\mathrm{S} \\
(\%)\end{array}$ & $\begin{array}{c}\text { DWD + } \\
(\%)\end{array}$ & $\begin{array}{c}\text { DWD } \\
(\%)\end{array}$ & $\begin{array}{c}\text { SD } \\
(\%)\end{array}$ \\
\hline & & & & & & & & & & & \\
\hline AP D65 & 0.31 & 0.33 & & & & & & & \\
\hline $\mathbf{1}$ & 0.16 & 0.10 & 470.30 & 81.86 & 0.16 & 0.10 & 469.70 & 82.90 & 10.71 & 0.00 & 1.27 \\
\hline $\mathbf{2}$ & 0.16 & 0.15 & 475.90 & 74.37 & 0.16 & 0.15 & 475.10 & 72.28 & 15.38 & 14.29 & 2.81 \\
\hline $\mathbf{3}$ & 0.16 & 0.20 & 481.10 & 66.15 & 0.17 & 0.20 & 481.20 & 62.66 & 1.89 & 1.92 & 5.28 \\
\hline $\mathbf{4}$ & 0.17 & 0.26 & 486.40 & 56.92 & 0.18 & 0.25 & 485.80 & 55.38 & 12.77 & 11.32 & 2.71 \\
\hline $\mathbf{5}$ & 0.18 & 0.31 & 491.10 & 47.93 & 0.19 & 0.32 & 491.30 & 45.86 & 4.17 & 4.26 & 4.32 \\
\hline $\mathbf{6}$ & 0.20 & 0.36 & 495.90 & 39.51 & 0.21 & 0.36 & 496.90 & 35.31 & 14.71 & 20.83 & 10.62 \\
\hline $\mathbf{7}$ & 0.22 & 0.41 & 502.70 & 30.85 & 0.22 & 0.40 & 503.50 & 28.49 & 6.50 & 11.76 & 7.64 \\
\hline $\mathbf{8}$ & 0.25 & 0.45 & 515.00 & 25.05 & 0.26 & 0.43 & 517.00 & 22.00 & 9.95 & 16.26 & 12.18 \\
\hline $\mathbf{9}$ & 0.27 & 0.48 & 535.10 & 33.74 & 0.27 & 0.48 & 535.30 & 35.20 & 1.23 & 1.00 & 4.32 \\
\hline $\mathbf{1 0}$ & 0.31 & 0.50 & 551.40 & 49.06 & 0.31 & 0.50 & 551.30 & 49.99 & 1.25 & 0.61 & 1.90 \\
\hline $\mathbf{1 1}$ & 0.35 & 0.52 & 559.40 & 62.16 & 0.35 & 0.51 & 559.40 & 62.61 & 0.00 & 0.00 & 0.73 \\
\hline $\mathbf{1 2}$ & 0.36 & 0.51 & 561.80 & 62.76 & 0.36 & 0.49 & 561.50 & 58.44 & 10.71 & 12.50 & 6.89 \\
\hline $\mathbf{1 3}$ & 0.37 & 0.49 & 564.60 & 61.67 & 0.37 & 0.49 & 564.00 & 62.02 & 16.67 & 13.89 & 0.57 \\
\hline $\mathbf{1 4}$ & 0.39 & 0.48 & 568.20 & 62.03 & 0.39 & 0.48 & 568.20 & 62.24 & 0.00 & 0.00 & 0.33 \\
\hline $\mathbf{1 5}$ & 0.40 & 0.46 & 571.10 & 61.01 & 0.40 & 0.46 & 571.10 & 63.84 & 0.00 & 0.00 & 4.63 \\
\hline $\mathbf{1 6}$ & 0.41 & 0.44 & 575.20 & 60.83 & 0.42 & 0.44 & 575.50 & 62.54 & 9.68 & 7.32 & 2.81 \\
\hline $\mathbf{1 7}$ & 0.43 & 0.43 & 578.30 & 60.88 & 0.43 & 0.43 & 578.10 & 63.52 & 4.65 & 6.45 & 4.34 \\
\hline $\mathbf{1 8}$ & 0.44 & 0.41 & 582.60 & 60.49 & 0.43 & 0.40 & 582.20 & 56.07 & 11.76 & 9.30 & 7.31 \\
\hline $\mathbf{1 9}$ & 0.45 & 0.40 & 586.00 & 60.18 & 0.46 & 0.40 & 586.20 & 61.29 & 6.25 & 5.88 & 1.84 \\
\hline $\mathbf{2 0}$ & 0.46 & 0.39 & 589.20 & 58.87 & 0.48 & 0.39 & 589.70 & 65.30 & 15.63 & 15.62 & 10.92 \\
\hline $\mathbf{2 1}$ & 0.47 & 0.37 & 592.40 & 57.90 & 0.46 & 0.37 & 592.80 & 56.15 & 0.00 & 12.50 & 3.03 \\
\hline
\end{tabular}

TABLE 2 Chromaticity values ( $x, y$ ), Dominant Wavelength (DW) in nanometers (nm) and Saturation percentage (S \%) for the measured FU vials and filter combinations. The percent difference in dominant wavelength (DWD $\pm \%$ ) and in saturation (SD \%). The chromaticity values of the achromatic point (AP) for the D65 illuminant are also presented. 


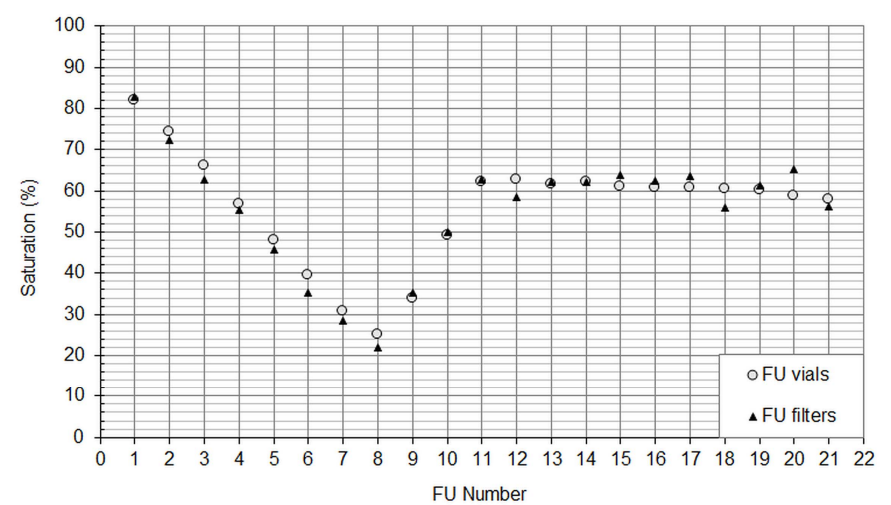

FIC. 5 Saturation percentage of the instrumentally measured vials and filter combinations.

tively). The specific DW and S values for each FU number are summarised in Table 2.

During the spectral measurements, it was noticed that the order of the filters affected the dominant wavelength, hence it is important to place the filters in the same order as in Table 1, the first filter specified on top, and the last at the bottom.

A total of 5 measurements were conducted for each vial and filter combination shown in Figure 3. From these, the standard deviation between measurements of each sample was established to be $\pm 0.1 \mathrm{~nm}$ for the dominant wavelength and \pm 0.45 $\%$ for the saturation measurements. The standard deviation for chromaticity coordinates $(x, y)$ measurements was \pm 0.003 . Similar results were obtained when identical tests were conducted using the D75 light source. Just as in the case of D65 conditions, higher DW deviations were obtained for FU6-8 and FU12-13 colours under the D75 illuminant, but always below $20.83 \%$. Then, the visual colour match between vials and filters was satisfactory, since the colours of the filters appeared visually identical to the vials when placed side by side inside the cabinet and observed under the D75 lamp.

The Munsell specifications corresponding to the FU vials are presented in Table 3. Both the values estimated visually under the D65 illuminant and the calculated values are provided.

\section{PROTOCOL FOR THE ASSEMBLAGE OF THE SCALE AND ITS USE}

The filters should be cut with a rectangular shape of dimensions $85 \times 10 \mathrm{~mm}$. The white frame, with a white background for the filters, could be of Perspex (Acrylox), PVC material or white painted wood; it should be as white as possible and not too glossy. The frame should have an A4 format $(297 \times 210 \mathrm{~mm})$ and the $21 \mathrm{FU}$ colours should be displayed in two rows, unlike the original scale that was divided in two parts (Figure 6). There should be a spacing in between the filters and the white background of $0.4 \mathrm{~mm}$. In this case, white pins were used to hold the filters to the frame, but any other

\begin{tabular}{|c|c|c|}
\hline FU Number & \multicolumn{2}{|c|}{ Munsell H V/C } \\
\hline & $\begin{array}{l}\text { Visual Measurement } \\
\text { D65 illuminant }\end{array}$ & Calculated \\
\hline 1 & $6.25 \mathrm{~PB} 3 / 12$ & 6.55 PB $3.38 / 17.8$ \\
\hline 2 & 5 PB 3/10 & 4.66 PB 2.92/10.8 \\
\hline 3 & $10 \mathrm{~B} 3 / 8$ & 9.14 B $2.55 / 7.0$ \\
\hline 4 & $2.5 \mathrm{~B} \mathrm{3/8}$ & 1.67 B 3.96/6.7 \\
\hline 5 & $7.5 \mathrm{BG} 3 / 8$ & 5.24 BG $2.20 / 6.2$ \\
\hline 6 & $2.5 \mathrm{BG} 3 / 8$ & 1.4 BG 2.21/6.5 \\
\hline 7 & $10 \mathrm{G} 3 / 8$ & 6.89 G $2.27 / 6.6$ \\
\hline 8 & $5 \mathrm{G} 4 / 8$ & 4.24 G 2.35/6.8 \\
\hline 9 & $2.5 \mathrm{G} 4 / 8$ & 1.08 G 2.92/6.9 \\
\hline 10 & 10 GY $5 / 8$ & 8.92 GY $3.47 / 6.9$ \\
\hline 11 & 7.5 GY 6/10 & 6.82 GY $4.16 / 7.5$ \\
\hline 12 & 6.25 GY $6 / 10$ & 6.11 GY $3.96 / 6.8$ \\
\hline 13 & 5 GY 5/8 & 5.12 GY $3.78 / 6.1$ \\
\hline 14 & 2.5 GY 5/8 & 2.95 GY $3.65 / 5.3$ \\
\hline 15 & $10 Y 5 / 8$ & 0.88 GY $3.43 / 4.7$ \\
\hline 16 & $7.5 Y 4 / 6$ & 7.31 Y $3.26 / 4.3$ \\
\hline 17 & $2.5 Y 4 / 6$ & 4.35 Y $3.21 / 4.2$ \\
\hline 18 & $10 \mathrm{YR} 4 / 4$ & 0.76 Y $2.88 / 4.2$ \\
\hline 19 & $7.5 \mathrm{YR} 3 / 6$ & 8.34 YR $2.74 / 4.3$ \\
\hline 20 & $5 \mathrm{YR} 3 / 6$ & 6.25 YR $2.47 / 4.2$ \\
\hline 21 & $2.5 \mathrm{YR} 3 / 4$ & 4.59 YR $2.25 / 4.2$ \\
\hline
\end{tabular}

TABLE 3 Munsell notations corresponding to FU numbers, estimated visually and calculated from the FU tristimulus values, through the 'colour2drop' software and the ASTM D1535 publication. The notations are specified as Hue (H), Value (V) and Chroma (C).

secure method could be used. As mentioned previously, the order of the filters, as shown in Table 1 is important.

The Modern FU scale should be used to estimate the colour of the water together with a Secchi disk [20] (white disk of approx. $30 \mathrm{~cm}$ in diameter attached to a rope), in the same way as the original scale developed by Forel and Ule was used. The Secchi disk is lowered vertically until it disappears from sight, this is the Secchi disk depth. Next, to determine the FU number, the disk is lifted to half this depth and the scale is held above the submerged Secchi disk. The operator then matches the colours of the scale filters in front of the white background, to the colour observed on top of the submerged Secchi disk, through one of the observation windows next to each FU plastic filters (Figure 6). To reduce the effect of direct sunlight's reflection on the filters, the operator should stand in the shadow or under a black umbrella, as Forel himself advised.

\section{DISCUSSION}

The industrial production of standardised plastic filters has provided the means to construct a simple colour comparator scale for natural waters. In this article, we have provided the recipe to combine filters so that they closely mimic the original FU scale. The plastic filters have the advantage that the colour is identical for all positions, while the thickness of the vial and the glass itself have an effect on the colour perception [28]. The glass produces differences in tristimulus values when measured at slightly different angles, so even if the colour of the objects was matched by visual comparison, the measurements 
a)

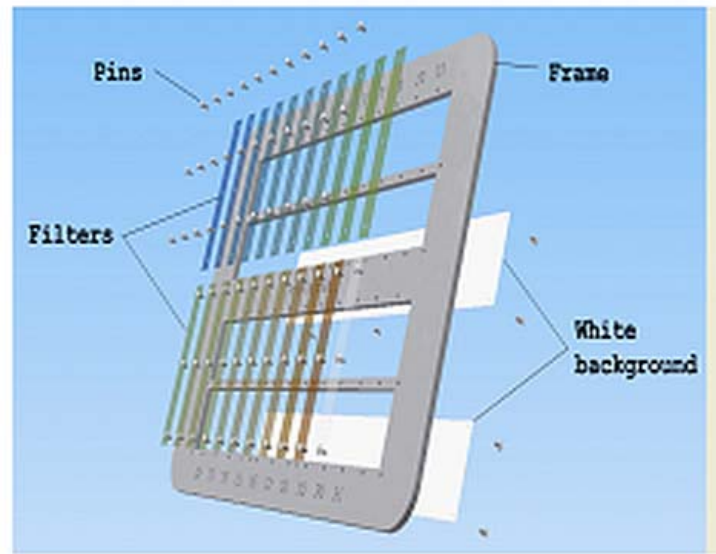

b)

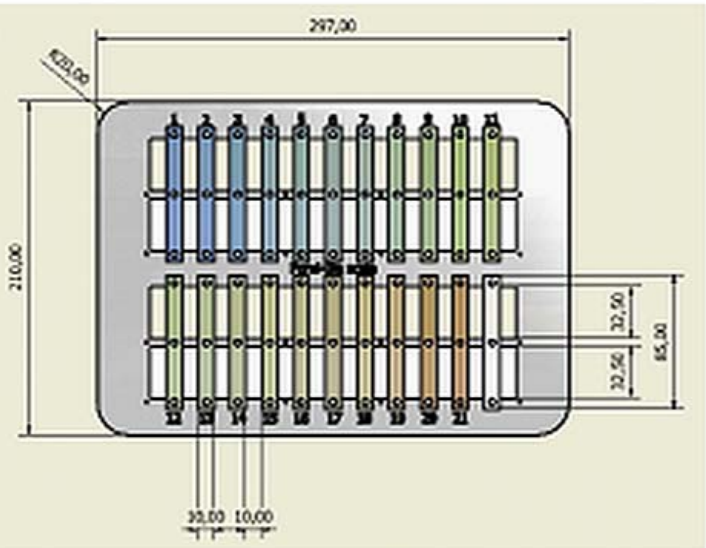

c)

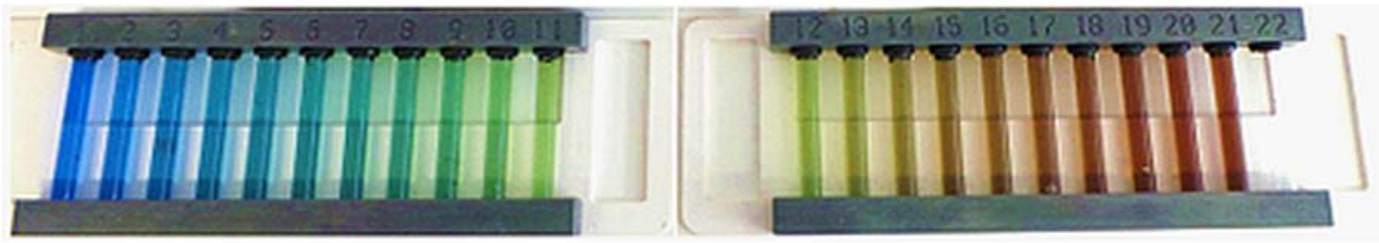

FIG. 6 Diagrams of the Modern FU plastic scale. The dimensions correspond to a format $\mathrm{A}_{4}(297 \times 210 \mathrm{~mm})$. There should be a spacing of $0.4 \mathrm{~mm}$ between the filters and the white background; c) Original Forel-Ule scale composed of coloured liquids in vials.

did not provide an exact chromaticity $(x, y)$ match. For that reason, the measurements had to be conducted at the exact same angle and position, for both the vials and the filters. The most stable position for the spectral measurements is achieved by placing the objects flat on the bottom of the cabinet, since a very slight horizontal or vertical shift of the measurement angle would output different values. The spectral measurement of both, filters and vials, provided a basis for the instrumental match between them. The measurement of the dominant wavelength (DW) of these objects (Figure 4) determined their hue while the saturation (S) (Figure 5) provided information on the intensity of the hue. A maximum $20.83 \%$ deviation in DW and $12.18 \%$ in saturation between vials and filters were considered satisfactorily accurate, since the colours of the matching FU number (vial and filter) appeared visually identical under the controlled conditions inside the cabinet.

We observed that the angle spacing between the different FU colours is not constant for the entire scale, in the bluer part of the scale (FU1-11) the average spacing is greater than in the browner part of it (FU12-21), providing less room for error and making it more difficult to exactly match the colours of the filters to the vials in this second part of the scale (Figure 4). Particularly, the DW spacing noticeably decreased between the FU16 and FU21 (Table 2), and, in the case of FU20, there is a DW difference of $3.5 \mathrm{~nm}$ with respect to the previous adjacent colour, FU19. This caused a greater difficulty to find closely a matching filter combination for this colour, and therefore a greater DWD. In the case of the FU6 color-match, the DWD resulted to be the highest $(20.83 \%)$ of all, simply because a filter combination with a closer chromaticity could not be found after numerous trials. A problem was encountered as well for FU 12 and FU13. The chromaticity coordinates of these two vials are very near, as shown in Table 2, indicating a greater difficulty in discriminating them. Although the deviations are still smaller than the relative changes in DW and $S$ between these two FU numbers, the DWD \% between vials and filters is the highest together with FU6 and FU20, when compared to the other matches.

Regarding the saturation, there is also a clear variation between FU numbers that also created difficulties when selecting the most accurate filter combinations. In some cases, such as FU6, an exact hue (DW) match was obtained between vials and specific filter combinations, but the saturation was much higher or lower, so the present filter combination for this colour was selected.

Two types of light sources, D65 and D75, were chosen to conduct colour-matching measurements, which correspond to two types of daylight simulators commonly used in colourmatching tests. The results showed that the colours were stable under these two different illuminations, since the colours of the selected filters matched the colours of the vials when inspected visually under the two types of illuminants (D65 and D75) in the lighting cabinet. Also, when comparing the filter combinations selected to the adjacent filter combinations (FU11 or FU14) they appeared to be obviously different in colour, proving that in case of doubt, the right colour would be selected by an observer. It was also considered to match the colours of the vials and filters under a D50 illuminant, also called 'horizon light'. It is a near white light source used in 
the evaluation of graphic arts and imaging applications, with similar amounts of red, green, and blue energy and it neither accentuates nor subdues colour. However, it has been related to the sunrise and the sunset light [34], a period of the day in which this scale should not be used. During twilight, the light intensity and spectral properties can change rapidly, affecting the colour of the water [40]. Colour-matching measurements of natural waters using this scale should be conducted at a solar zenith angle above $60^{\circ}\left(90^{\circ}\right.$ - zenith; $0^{\circ}$ - horizon), as it has been shown that the sun angle affects the water-leaving radiance [41, 42]. This is also to ensure enough light is available and that the measurements are conducted under comparable light conditions, corresponding with the D65 CIE standard illumination, which represents the average daylight.

The final objective of this study was to reproduce the colours of the vials as identically as possible using the lighting filters, so that an observer would select the same colour in both formats when looking at a body of water. The authors can positively state that this objective was achieved, since when inspecting the filter-vial combinations individually and all together, obvious colour-matches between the correspondent vials and filters could be made. The colours of the selected filter combinations were certainly different between them, but matching the colours of each corresponding vial.

In general, the Munsell values calculated matched closely the values estimated visually, but in some cases the lightness values $(\mathrm{V})$ and the chroma $(\mathrm{C})$ of the chips were higher when estimated visually, compared to the calculations made using the reflectance spectra. In general, the hue values remained similar, the closest chip value available in the book with respect to the calculated values were selected. Except for FU12, where neither of the Munsell chips available from the 'Munsell book' exactly matched its colour. There was an obvious visual colour difference between FU12 and FU13, so the FU12 colour was estimated to be allocated in between 7.5 GY 6/10 and 5 GY 6/10, since the colour of the vial (FU12) fell right in between both of these chips when compared simultaneously. For that reason, the Munsell value presented in Table 3 for FU12 (6.25 GY 6/10) was not actually determined visually, but deduced. Also, the 'chroma' of FU1 did not completely match the chips available in the book, the maximum chroma value available was 12 , but it could be inferred that a higher values was necessary to match the colour of the vial. These differences between calculated values from instrumental and visual measurements were also encountered by Davies-Colley et al. $(1988,1997)[22,43]$, who attributed these differences to the difficulty of interpolation between widely-spaced saturation levels for each hue. Since this is a scale meant to compare colours by means of the human eye, we recommend to use the values estimated visually rather than mathematically.

Most of the Munsell values attributed to the FU indexes, coincide with Munsell specifications found in natural waters according to the chromaticity coordinates and the Munsell specifications published [22], [43]-[45]. The interesting part about connecting the FU scale to the Munsell system is to include additional information in monitoring the water environment. For example, the Munsell system is composed of more hues than the FU scale. Also, the FU index provides information on variable hues (blue to brown, FU1 to FU21), but only provides one specific saturation value for each FU index. The Munsell system provides variable saturation values for the same hue, being able to distinguish between whitish blue, greyish blue or pure blue. For example, the whitish content in the water caused by a particular type of sediment or organism (e.g. chalk, coccoliths) does not affect the hue, but affects the saturation. In addition, the variability of the saturation from the Munsell system, as well as the brightness component (V), can help the observer better match the colour of the water body. The inclusion of these two components will be of special interest for the development of a smartphone application, as part of the Citclops project (www.citclops.eu), based on the same principle of colour comparison as the FU scale, but using digital images. The user could scroll down the different hues as well as different lightness values and chromas to more accurately match the colour of a picture taken by a smartphone.

In any case, the hue component remains the most important feature of water colour, since it has been proven to be correlated with chlorophyll-a concentration and CDOM absorbance [4], and has been acknowledged to be the most important colour attribute that can be accurately specified [22]. In fact, the different hues of the water are used by governmental agencies to define the quality of bathing waters based on hues and have also established that waters should not change by more than 5 points on the Munsell scale.

To get a reliable natural water colour comparator scale, the recommendations for the assemblage stated in this document should be followed. Hence, the frame of the scale should be of the recommended size, since a previous prototype of the scale (1/2 of an A4) complicated the colour comparison in the field. The background of the filters has to be white, over air, so that the colour does not differ from the original FU scale. The filters should be in the right order, as minor changes in dominant wavelengths could be measured when this order was altered. The filters should also be placed as tight and flat as possible, avoiding any bending, as it could complicate the reading. Filters are made of a highly reflective plastic, making it difficult to see the colour when observed under direct sunlight, for that reason the observations should be achieved in the shade, as recommended by the traditional FU measurement protocols [46].

The Citclops consortium plans to initially manufacture 50 of these scales and freely distribute them among specific environmental volunteering groups. Depending on the success of this initiative, more scales will be manufactured and perhaps distributed as a kit, constituted by a frame and all the cut and numbered filters. The user would only need to assemble all the parts. To give the reader an idea of the costs to manufacture a single sacle: 1 coloured filter sheet (Lee, $0.5 \times 1.2 \mathrm{~m}$ ) costs 10 Euro, a total of 30 different colour effect filter sheets are required, and each sheet can be cut into approximately 100 parts of $10 \times 85 \mathrm{~mm}$ (so each part costs around 0.1 Euros). Then, a total of 52 filter parts are necessary for each scale (some colour parts are used for several FU colours), thus, the cost of all the filters necessary for one scale totals 5.2 Euros. The frame was manufactured for around 40 Euros. The authors are in con- 
tact with possible professional manufacturers to facilitate the make of a low-cost Forel-Ule scale.

In addition, the Citclops consortium is working on the development of a smartphone application (or APP) that will include a digitalised version of the Forel-Ule scale. This application will make it easier for users to estimate the colour of the waters and provide more data to the consortium, since it could be distributed easily and at no cost. Work is been undertaken on the transformation of the FU colours of the original scale to a digital format using similar colorimetric methods to the ones used in this document. A first 'APP' prototype was developed, but the details on this work are beyond the scope of this investigation. Due to the different smartphone screen displays and camera characteristics, the plastic FU scale could be used as a way of validating the colours selected by the users via the application. The APP includes an option where the user can introduce the FU number measured with the Modern FU scale, the comparison of both estimates (FU from the APP, and FU from the scale) would give information on the accuracy of the digital colours.

\section{CONCLUSIONS}

This manuscript presents specifications on the development of a 'Do-it-Yourself' Forel-Ule scale, manufactured using lighting filters and a white frame. This modern scale is an improvement with respect to the original scale developed by the creators Forel and Ule, and other commercially available scales, as it is light-weighted, inexpensive and easy to produce. It is also safe (no chemicals nor breaking glass) to assemble and use by observers of any age, willing to participate in water quality monitoring. The filter combinations selected match accurately the colours estimated through spectral reflectance measurements of the FU vials and visual comparisons, undertaken in a controlled environment. The laboratory set-up and the protocols followed for the colourmatching exercises are commonly used in the industry to determine colours under different illuminations and to match colours of objects. The filters employed are standardised, highly stable (able to stand high temperatures) and not prone to colour change over time. The format of the scale was specifically designed to easily estimate the colours of natural waters by anyone without colour vision deficiencies, using a Secchi disk. The FU numbers are expressed also as Munsell colour notations, a system used by governmental agencies for monitoring water quality. These notations were measured in the laboratory and calculated from reflectance measurements. The authors foresee as future research the association of chlorophyll-a concentration, CDOM absorbance and TSM concentration with these colour indexes, FU and Munsell. In this way, the FU index could be used by local and governmental authorities to determine the quality of water bodies at a local and global scale.

\section{ACKNOWLEDGEMENTS}

The authors wish to thank Jasper Hofstede of Controllux B.V. (The Netherlands), the provider of the colour effect filters, for his help on selecting the filters. We like to thank Edwin Keijzer, Roel Bakker and Johan van Heerwaarden, highly skilled engineers of the instrumental department of the Netherlands Institute for Sea Research and responsible for the FU frame design and first filter cuttings. The research described in this paper is partly supported by the Citclops European project (FP7-ENV-308469; www.citclops.eu). The opinions expressed in this paper are those of the authors and are not necessarily those of Citclops projects partners or the European Commission.

\section{References}

[1] W. Ule, "Die Bestimmung der Wasserfarbe in den Seen, Kleinere Mittheilungen," Dr. A. Petermanns Mitth. aus Justus. Perthes Geogr. Anstalt 38(70)-(71) (1892).

[2] G. E. Hutchinson, A treatise on limnology. Vol. I, Geography, physics, and chemistry (John Wiley At Sons, Haboken, 1957).

[3] F. A. Forel, Le Léman, Monographie Limnologique II (Librairie de l'Université, Lausanne, 1904).

[4] M. R. Wernand, H. J. Van Der Woerd, and W. W. C. Gieskes, "Trends in Ocean Colour and Chlorophyll Concentration from 1889 to 2000, Worldwide," PLOS One 8(6), (2013).

[5] Y. Huot, M. Babin, F. Bruyant, C. Grob, M. S. Twardowski, and H. Claustre, "Relationship between photosynthetic parameters and different proxies of phytoplankton biomass in the subtropical ocean," Biogeosciences 4, 853-868 (2007).

[6] IOCCG, "Remote Sensing of Ocean Colour in Coastal, and Other Optically-Complex Waters," in Reports of the International Ocean Colour Coordinating Group No. 3, S. Sathyendranath, ed., p. 144 (IOCCG, Darmouth, 2000).

[7] J. T. 0. Kirk, "Optical water quality, what does it mean and how should we measure it?," Water Pollut. Control 60, 194-197 (1988).

[8] D. G. Smith, G. F. Croker, and K. McFarlane, "Human perception of water appearance," New Zeal. J. Mar. Fresh. 29(1), 29-43 (1995).

[9] D. G. Smith, A. M. Cragg, and G. F. Croker, "Water clarity criteria for bathing waters based on user perception," J. Environ. Manage. 33(3), 285-299 (1991).

[10] P. A. Del Giorgio, and R. H. Peters, "Patterns in planktonic P:R ratios in lakes: Influence of lake trophy and dissolved organic carbon," Limnol. Oceanogr. 39(4), 772-787 (1994).

[11] D. L. Christensen, S. R. Carpenter, K. L. Cottingham, S. E. Knight, N. LeBouton, J. P. Schindler, D. E. Voichick, et al., "Pelagic responses to changes in dissolved organic carbon following division of a seepage lake," Limnol. Oceanogr. 41, 553-559 (1996).

[12] B. Wissel, W. J. Boeing, and C. W. Ramcharan, "Effects of water color on predation regimes and zooplankton assemblages in freshwater lakes," Limnol. Oceanogr. 48(5), 1965-1976 (2003).

[13] L. M. Juarez, K.-H. Holtschmit, J. J. Salmeron, and M. K. Smith, "The effects of chemical and visual communication, space availability, and substratum color on growth of the juvenile freshwater prawn Macrobrachium rosenbergii (De Man)," J. Exp. Mar. Bio. Ecol. 110(3), 285-295 (1987).

[14] A. Kullberg, "Benthic macroinvertebrate community structure in 20 streams of varying $\mathrm{pH}$ and humic content," Environ. Pollut. 78(1)-(3), 103-106 (1992). 
[15] T. A. Haines, V. T. Komov, V. E. Matey, and C. H. Jagoe, "Perch mercury content is related to acidity and color of 26 Russian lakes," Water Air Soil Poll. 85, 823-828 (1995).

[16] A. Nilsson, and L. Håkanson, "Relationships between mercury in lake water, water colour and mercury in fish," Hydrobiologia 235/236, 675-683 (1992).

[17] J. B $\emptyset$ gestrand, P. Kristensen, and B. Kronvang, "Source apportionment of nitrogen and phosphorus inputs into the aquatic environment," Report 7, 48 (European Environment Agency, Copenhagen, 2005).

[18] D. R. J. Moore, P.-Y. Caux, “Ambient Water Quality Criteria for Colour in British Columbia: Technical Appendix" Ministry of Environment. Water Quality Branch (1997). http://www.env.gov.bc.ca/wat/wq/BCguidelines/colour

[19] W. Preisendorfer, "Secchi disk science: Visual optics of natural waters," Limnol. Oceanogr. 3, 909-926 (1986).

[20] P. A. Secchi, "Relazione delle esperienze fatte a bordo della pontificia pirocorvetta l'Immacolata concezione per determinare la trasparenza del mare," II Nuovo Cimento 20, 205-238 (1864).

[21] I. D. Cuthbert, and P. a. Del Giorgio, "Toward a standard method of measuring color in freshwater," Limnol. Oceanogr. 37(6), 13191326 (1992).

[22] R. J. Davies-colley, Smith, D.G, D. J. Speed, and J. W. Nagels, "Matching natural water colors to Munsell standards," J. Am. Water Resour. As. 33(6), 1351-1361 (1997).

[23] R. J. Davies-Colley, W. N. Vant, and D. G. Smith, Colour and Clarity of Natural waters (Ellis Horwood Limited, Chicheste, 1993).

[24] T. Y. Crowell Company, The Science of Color (Optical Society of America, Washington D. C, 1966).

[25] A. H. Munsell, "A Pigment Color System and Notation," Am. J. Psychol. 23, 236-244 (1912).

[26] R. J. Davis-Colley, and D. Ballantine "Suitability of NZ Rivers for contact recreation," NIWA, National Institute of Water and Atmospheric Research, Report 133, 19 (2010).

[27] Ministry for the Environment Manatu Mo Te Taiao, "Guidelines for the Management of Water Colour and Clarity, No. 2," p. 77 (1994).

[28] S. Novoa, M. R. Wernand, and H. J. van der Woerd, "The ForelUle scale revisited spectrally: preparation protocol, transmission measurements and chromaticity," J. Europ. Opt. Soc. Rap. Public 8,13057 (2013).

[29] D. Lison, M. De Boeck, V. Verougstraete, and M. Kirsch-Volders, "Update on the genotoxicity and carcinogenicity of cobalt compounds," Occup. Environ. Med. 58(10), 619-625 (2001).

[30] J. H. Jerome, R. P. Bukata, P. H. Whitfield, and N. Rousseau, "Colours of natural waters: 1 . Factors controlling the dominant wavelength," Northwest Sci. 68, 43-52 (1994).

[31] J. H. Jerome, R. P. Bukata, P. H. Whitfield, and N. Rousseau, "Colours of natural waters: 2 . Observations of spectral variations in British Columbia," Northwest Sci. 68, 53-64 (1994).
[32] R. S. Berns, "Principles of color technology," F. W. Billmeyer, and M. Saltzman, ed., 247 (John Wiley ¿t Sons, Haboken, 2000).

[33] D. Farnsworth, "The Farnsworth-Munsell 100-Hue and Dichotomous Tests for Color Vision," J. Opt. Soc. Am. 33, 568-574. (1943).

[34] HunterLab, "Equivalent White Light Sources and CIE Illuminants," Appl. Notes 17(5), 1-5 (2008).

[35] ASTM Standard E1164, "Standard Practice for Obtaining Spectrometric Data for Object-Color Evaluation," American Society for Testing and Materials (ASTM) International (2003).

[36] ASTM Standard E179-12, "Standard Guide for Selection of Geometric Conditions for Measurement of Reflection and Transmission Properties of Materials," American Society for Testing and Materials (ASTM) International (2012).

[37] ASTM Standard E308, "Standard Practice for Computing the Colors of Objects by Using the CIE System," American Society for Testing and Materials (ASTM) International (2013).

[38] G. Wyszecki, and W. S. Stiles, Colour Science: Concepts and Methods, Quantitative Data and Formulae (John Wiley a Sons, Haboken, 1982).

[39] ASTM Standard D1535, "Standard Method of Specifying Color by the Munsell System," American Society for Testing and Materials (ASTM) International (2013).

[40] W. N. McFarland, and F. W. Munz, "Part II: The photic environment of clear tropical seas during the day," Vision Res. 15(10), 10631070 (1975).

[41] J. L. Mueller, G. S. Fargion, C. R. Mcclain, J. L. Mueller, R. R. Bidigare, C. Trees, W. M. Balch, et al., "NASA/TM-2003- Ocean Optics Protocols For Satellite Ocean Color Sensor Validation," Revision 5, Volume V: Biogeochemical and Bio-Optical Measurements and Data Analysis Protocols NASA (2003). http://oceancolor.gsfc.nasa.gov/

[42] M. Wang, "Effects of ocean surface reflectance variation with solar elevation on normalized water-leaving radiance," Appl. Optics 45(17), 4122-4128 (2006).

[43] R. J. Davies-Colley, W. N. Vant, and R. J. Wilcock, "Lake water color: Comparison of direct observations with underwater spectral irradiance," Water Resour. Bull. 24(1), 11-18 (1988).

[44] S. Q. Duntley, R. W. Austin, W. H. Wilson, C. F. Edgerton, and S. E. Moran, Ocean Color Analysis (NTiS 7420, 1974).

[45] R. C. Smith, and K. S. Baker, "Optical classification of natural waters," Visibility Laboratory. Scripps Institution of Oceanography. La Jolla, California., p. 20 (1977). http://misclab.umeoce.maine.edu/education/VisibilityLab/ reports/SIO_77-4.pdf

[46] M. R. Wernand, Poseidon's paintbox historical archives of ocean colour in global change (PhD Thesis, Utrecht University, 2010). 